\title{
Parametric Optimization of Ares I Propellant Slosh Characteristics Using Frequency Response Criteria
}

\author{
Jeb S. Orr ${ }^{*}$ \\ Science Applications International Corporation, Huntsville, AL 35802 \\ Charles E. Hall ${ }^{\dagger}$ \\ NASA Marshall Space Flight Center, Huntsville, AL 35802
}

\begin{abstract}
[Abstract] A novel technique for developing propellant slosh damping requirements with respect to the stability characteristics of large flexible launch vehicles is presented. A numerical algorithm is devised which allows an automated software program to rapidly converge to pseudo-optimal solutions that minimize required propellant slosh damping for multiple tanks while maintaining constraints on the frequency response characteristics of a particular open-loop plant transfer function. An implementation of the algorithm using a high-order linear model of the Ares I plant dynamics considers all relevant dynamic interactions of flexible body modes, propellant slosh, and nozzle inertia effects. A high-resolution propellant damping requirements table is produced that can be used for baffle design. The method is demonstrated to provide exceptional speed and accuracy when compared with the alternative human-in-the-loop approach.
\end{abstract}

\section{Introduction}

The influence of sloshing propellant on the stability and control of large flexible booster rockets is a well known problem that has been extensively researched throughout various manned and unmanned launch vehicle programs. ${ }^{1,23}$ Excitation of liquid motion at its fundamental frequency can rapidly lead to an instability that renders the control system ineffective or results in excessive forces and moments that may compromise structural integrity. ${ }^{4}$ At the minimum, an unstable closed-loop propellant mode will result in an attitude limit cycle that can overstress TVC subsystems or unnecessarily expend maneuvering propellant. ${ }^{5,6}$ It is of paramount importance that the liquid motion be well understood so that it can be appropriately stabilized or suppressed via active or passive techniques. ${ }^{7}$

A typical approach of the flight controls discipline is the use of a planar linear perturbation dynamics model of the vehicle that includes the coupled dynamics of the elastic modes and the sloshing propellant. ${ }^{8,9}$ The external forces due to nonlinear propellant sloshing of each $j$ of $n$ tanks is approximated by a linear spring-mass-damper confined to motion normal to the vehicle thrust axis. The spring-mass-damper model is appropriate for rockets with cylindrical tanks where the dominant acceleration is normal to the liquid's lateral degrees of freedom. Other sloshing models, such as the pendulum approximation, may be necessary for certain vehicle or container configurations.

The $2^{\text {nd }}$-order linear slosh dynamics are described by the equations

$$
\ddot{\delta}_{s j}+2 \zeta_{s j} \omega_{s j} \dot{\delta}_{s j}+\omega_{s j}^{2} \delta_{s j}=-\ddot{\delta}_{z}-l_{s j} \ddot{\varphi}-\sum_{i=1}^{k} \psi_{s i j} \ddot{\eta}_{i}, \quad j=1 \ldots n
$$

where $\delta_{s j}$ is the perturbation state of an equivalent mass $m_{s j}$ that approximates the portion of liquid that is involved in sloshing, $\varphi$ is the perturbation angle relative to the trajectory, and $\ddot{\delta}_{z}$ is the body lateral acceleration. The eigenvectors $\psi_{s i j}$ and the generalized accelerations $\ddot{\eta}_{i}$ represent forcing of the slosh dynamics via elastic motion. The non-sloshing fluid is assumed to be stationary in the body frame, and the equivalent natural frequencies $\omega_{s j}$ and viscous damping $\zeta_{s j}$ are nonlinear functions of the axial acceleration and the liquid and container properties. ${ }^{3}$ It is clear that the liquid motion is excited by rigid body translation and rotation as well as forces exerted by the elastic deflection of the vehicle at the tank nodes. The liquid response also drives both rigid body and

* Flight Controls Engineer, Advanced Technology Division, jeb.s.orr@saic.com

$\dagger$ Senior Aerospace Engineer, Flight Controls Branch, charles.e.hall@nasa.gov 
flexible modes. It is assumed that the perturbations are small and that the forcing function frequency content is sparse near the true natural frequency of the liquid motion. ${ }^{3}$

While it is possible to compute analytical stability criteria for reduced models of the launch vehicle dynamics, ${ }^{6}$ as multiple slosh and additional bending modes are included, the order of the model increases sufficiently to preclude an analytical solution. A numerical analysis of the frequency response characteristics of the high-order open-loop model can be utilized to assess the closed-loop stability with many of the relevant elements (actuator models, bending filters, and so on) included. Stability is characterized in terms of gain and phase margins in addition to other criteria that constrain open-loop response of the bending or sloshing modes. ${ }^{8}$

Sloshing propellant response is often neglected explicitly in the design of a controller and is passively suppressed using baffles or other mechanical devices. A necessary tradeoff is the sacrifice of injected mass to account for the increased mass of the baffle mechanisms versus additional control system complexity and risk associated with insufficient slosh damping. In the early design phases of the vehicle, the exact propellant damping characteristics and their interaction with the control system are largely unknown to the control designer. A baseline, conservative damping requirement may be specified, but must be iteratively modified as the vehicle design matures. The damping requirements at a particular liquid level may increase or decrease due to configuration changes or performance constraints, or the structure may not be able to accommodate a mechanical baffle at the specified liquid level. The ability to rapidly generate a damping requirements profile based on a fixed control system configuration can greatly accelerate the design lifecycle.

\section{Motivation}

Propellant slosh damping in the Ares I Crew Launch Vehicle (CLV) is of particular interest due to the potential for significant control-slosh interaction. The sloshing fluid is located far forward of the composite first stage center of mass, the vehicle is highly flexible, the axial acceleration profile varies greatly, and the vehicle and control system characteristics before and after staging are significantly different. The elastic deformation of the integrated stack during first stage ascent may couple with the liquid motion due to coalescence of the oxidizer slosh and first lateral bending mode frequencies at peak axial acceleration. Although the tank is nearly full and the liquid level is constant during first stage ascent, appropriate baffle placement is required to ensure gain stabilization of the oxidizer mode. An example of the time-varying nature of the slosh parameters is shown in Figure 1.
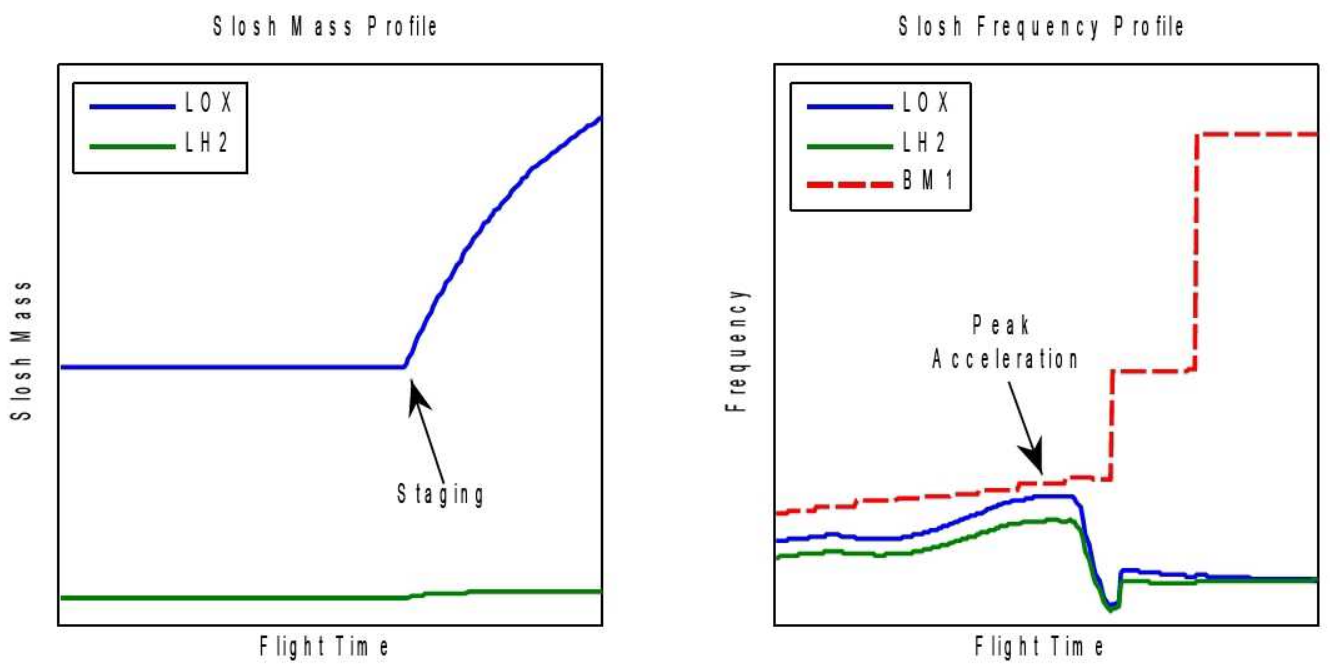

Figure 1: Typical slosh parameter variation

During upper stage flight, the shift in frequency and increase in sloshing propellant mass as the tanks empty preclude gain stabilization of the slosh dynamics. Both slosh modes appear at frequencies near the rigid-body control crossover frequency, and while phase stabilization of the slosh response is straightforward, it naturally leads 
to reduced phase margin if the response is underdamped. Most importantly, many of the system parameters have significant uncertainty.

Accurate determination of acceptable propellant slosh response as it relates to vehicle stability margins and performance objectives is a difficult task. An optimal damping profile varies with liquid level as the liquid and vehicle mass properties change, and is further complicated by the presence of two interacting tanks whose cross-coupling effects may be significant.

The compensated open-loop dynamics of the Ares I vehicle and similar configurations exhibit conditional stability at least in one dimension, and typically in both magnitude and phase. ${ }^{8}$ As such, a stability analysis can be readily visualized using a Nichols chart, where the log magnitude of the proper plant open-loop transfer function is plotted with respect to phase on a set of orthogonal axes. A natural choice of loop opening occurs at the actuator input command, where the system dynamics are SISO and the classical gain and phase margins relate directly to multiplicative input uncertainty and delay uncertainty in the autopilot loop. An example of a typical Nichols response is shown in Figure 2.

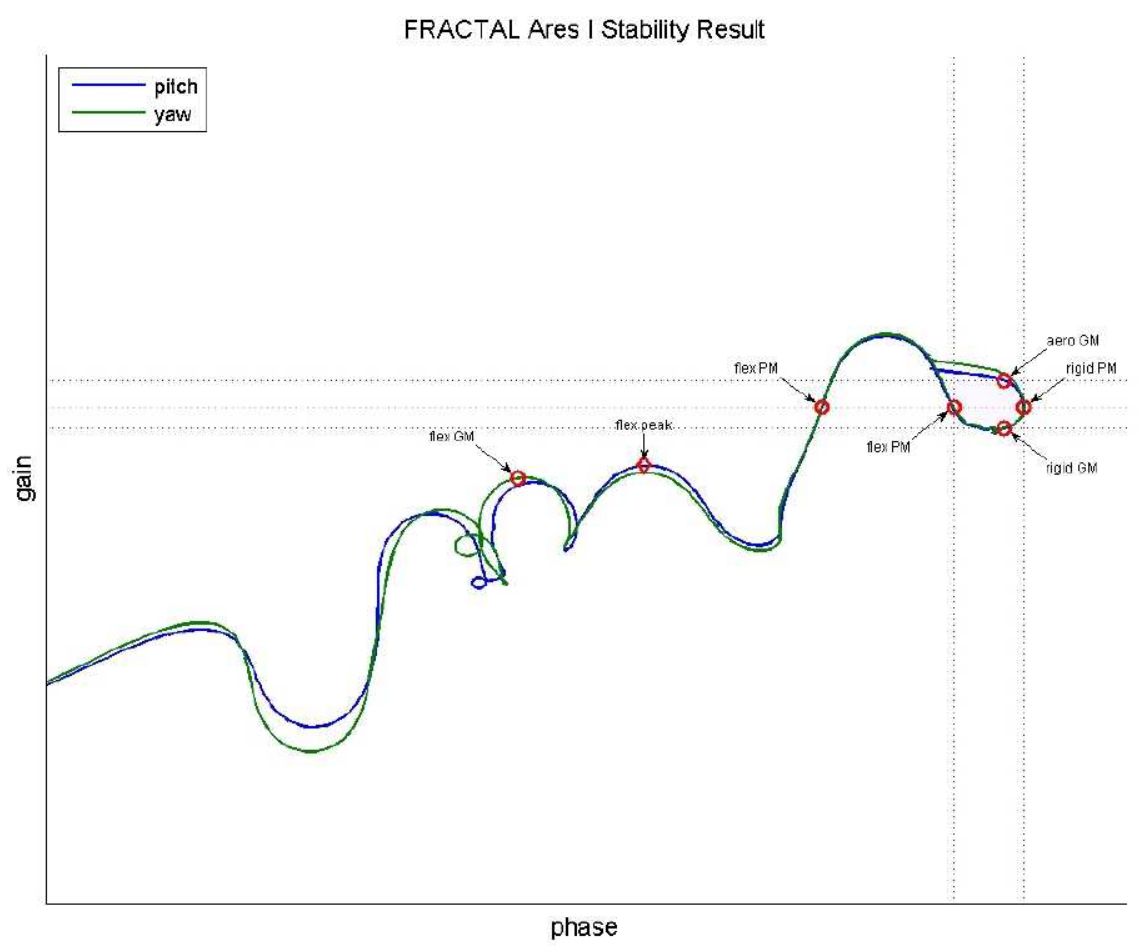

Figure 2: Nichols response example

The path through the resultant magnitude-phase space is traced in increasing frequency. The Nichols criteria are a direct mapping of the Nyquist criterion: knowledge of the location of the open loop poles and the number of encirclements of the critical point $-1+j 0$ can be used to assess relative stability of the closed-loop system dynamics. Multiple gain and phase margins are computed, each corresponding to the approximate frequency of the associated physical dynamics. Other robust frequency-domain stability criteria may be applied to the open-loop frequency response, including a limitation on the peak open-loop bending mode response, shown as "flex peak" above.

One approach to analyzing the stability of the closed-loop system utilizes the aforementioned baseline tank configuration. The robustness of the control system is determined by modifying system parameters either manually 
or via a Monte Carlo method. If it can be determined that stability objectives are not achieved either in the time or frequency domain, the damping is increased in the time vicinity of the problem area and the simulation is re-run.

This human-in-the-loop approach to the determination of propellant slosh damping requirements is slow and imprecise. A slightly more systematic approach allows an analyst to compare the frequency-domain trajectories of multiple systems with slightly varied damping characteristics. The analyst must select the best system response at the displayed time-slice. An example of this type of operator display appears in Figure 3. Clearly, the resolution is limited and the method is labor-intensive.

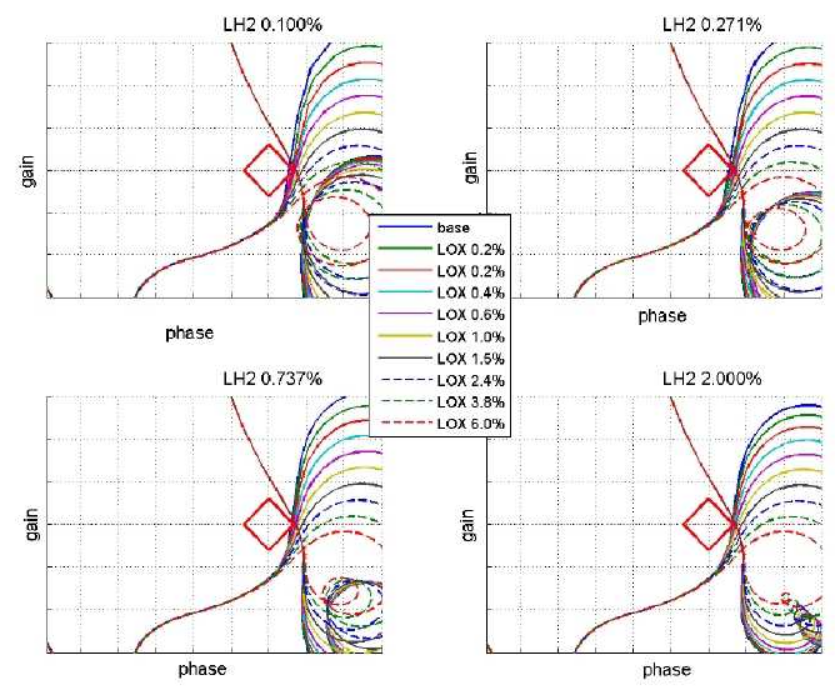

Figure 3: Manual damping determination method

Any automated search of the solution space must attempt to determine, within reasonable bounds and with sufficient fidelity, the correct values of damping ratios for each tank at each time in the trajectory that result in acceptable time and frequency domain control system performance. It has been determined via simulation that the solution region is only vaguely known, that multiple minima exist, and that the solution is highly sensitive within a very small region. The computational requirements are daunting if the solution is to be obtained via a direct search with good precision.

An efficient algorithm can capitalize on the ability of modern computer hardware to calculate the response of high-order linear dynamics models with exceptional speed as compared with computer hardware available for similar analyses in past launch vehicle programs. All dynamic characteristics can be considered, including multiple tanks and dozens of flexible modes, with minimal impact on computation rate. It is therefore possible to utilize high-order linear models within a specialized parameterization mechanism that can be used to automatically optimize various system parameters with greater speed and accuracy than possible with a human operator. The use of this capability in the design of a novel algorithm for optimizing propellant slosh damping requirements is the primary motivation of our approach.

\section{Problem Solution}

The proposed method of determining a near-optimal parameterization of the linear, time-invariant SISO system,

$$
\dot{\boldsymbol{x}}=\boldsymbol{A}(\boldsymbol{\theta}) \boldsymbol{x}+b u, \quad y=C \boldsymbol{x}
$$

where $\theta$ is a vector parameter, relies on a geometric interpretation of the complex frequency response that can be calculated as

$$
G(j \omega, \boldsymbol{\theta})=C[j \omega \boldsymbol{I}-\boldsymbol{A}(\boldsymbol{\theta})]^{-1} b .
$$

Let there be a function $v$ that maps the complex valued function $G(j \omega, \boldsymbol{\theta})$ onto the Nichols plane such that 


$$
v(G(j \omega, \boldsymbol{\theta}))=\left[\begin{array}{l}
\measuredangle G(j \omega, \boldsymbol{\theta}) \\
20 \log |G(j \omega, \boldsymbol{\theta})|
\end{array}\right]=\boldsymbol{\eta}(j \omega, \boldsymbol{\theta})
$$

which is the function of real frequency $\omega$ appearing in a Nichols plot. Suppose that the gradient of $\eta$ with respect to the parameters $\boldsymbol{\theta}$ is known approximately or can be estimated, that is, the directions of the $\omega$-trajectory perturbations with respect to the $k$ parameters of interest $\theta_{i}$ can be determined:

$$
\frac{\partial \boldsymbol{\eta}}{\partial \theta_{1}} \approx \boldsymbol{\phi}_{1}(j \omega), \frac{\partial \boldsymbol{\eta}}{\partial \theta_{2}} \approx \boldsymbol{\phi}_{2}(j \omega), \ldots, \frac{\partial \boldsymbol{\eta}}{\partial \theta_{k}} \approx \boldsymbol{\phi}_{k}(j \omega) .
$$

Intuitively, the functions $\phi_{i}$ point in the direction of the expected trajectory perturbation to be taken by the function $\boldsymbol{\eta}(j \omega, \boldsymbol{\theta})$ with respect to a small perturbation in $\theta_{i}$. The values $\boldsymbol{\phi}_{i}$ may be resolved, for example, into their magnitude and phase components along the frequency path. Intuitively, one may then prescribe convex regions $S_{i}$ in the Nichols plane whose boundaries have components that are normal to $\nabla_{\theta} \eta$. Thereafter, define a binary function

$$
\boldsymbol{\tau}(\boldsymbol{\eta}, \boldsymbol{S})=\left\{\begin{array}{l}
1, \boldsymbol{\eta} \not \subset S_{i}, \forall i \\
0, \text { otherwise }
\end{array}\right.
$$

which is presumably a binary test of the presence or absence of the intersection of the open-loop response $\eta$ subject to the parameterization $\boldsymbol{\theta}$ with the set of exclusion regions $\boldsymbol{S}$.

The binary function $\boldsymbol{\tau}$, subject only to perturbations of the parameters $\boldsymbol{\theta}$, is a straightforward test that may be incorporated into the following binary search algorithm, given initial guesses $\theta_{i, \max }[0], \theta_{i, \min }[0], i=1 \ldots k$ on the upper and lower bounds of each of the $k$ parameters. The algorithm is formulated as follows:

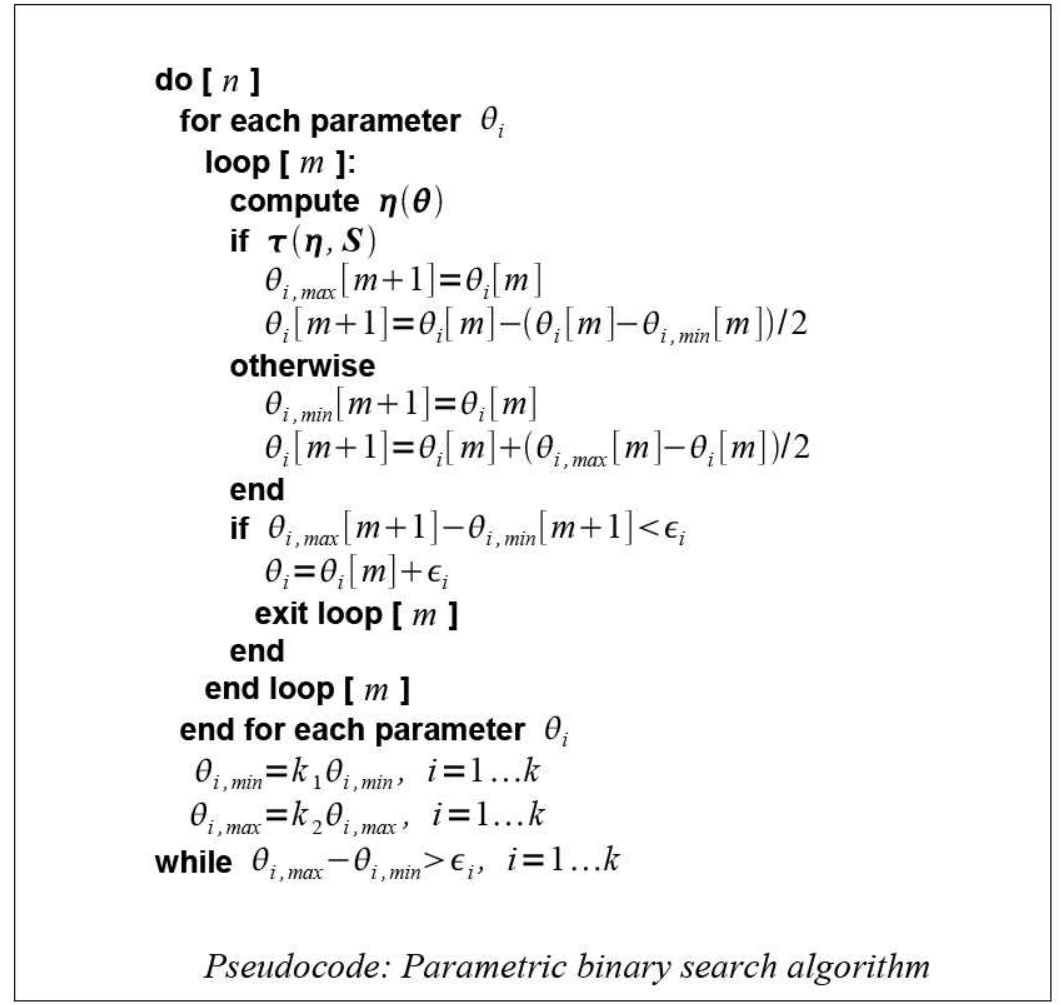

The algorithm progressively bounds the value of each parameter $\theta_{i}$ so as to constrain the solution set of parameters $\boldsymbol{\theta}$ (where $\boldsymbol{\tau}=1$ ) within the arbitrarily small region $\boldsymbol{\epsilon}$ near the boundaries of $S_{i}$. The algorithm is designed to converge efficiently to the semi-optimal values $\theta_{i}$ when only one unique region exists with respect to $\theta_{i}$ at the switching boundary of $\boldsymbol{\tau}$, and it is known that the set of trajectory perturbation vectors $\boldsymbol{\phi}_{i}(j \omega)$ are 
approximately aligned at similar $\omega$. Proper selection of the exclusion regions $S_{i}$, the initial bounds $\theta_{i, \min }[0], \theta_{i, \max }[0]$, and the convergence criteria $\epsilon_{i}$ govern the algorithm's efficiency.

A practical example applies the algorithm to a slosh response optimization problem. For a phase-stabilized slosh mode, it is postulated that the absolute magnitude of the open-loop gain shall not exceed some gain value $g_{\max }$. Therefore, the boundary of the region $S_{1}$ becomes the $g_{\max }$ line in the phase-magnitude plane, and the remaining boundaries of the region are placed such that phase uncertainty is tolerated. An example of the phasestabilized and gain-suppressed slosh exclusion region is shown in Figure 4.

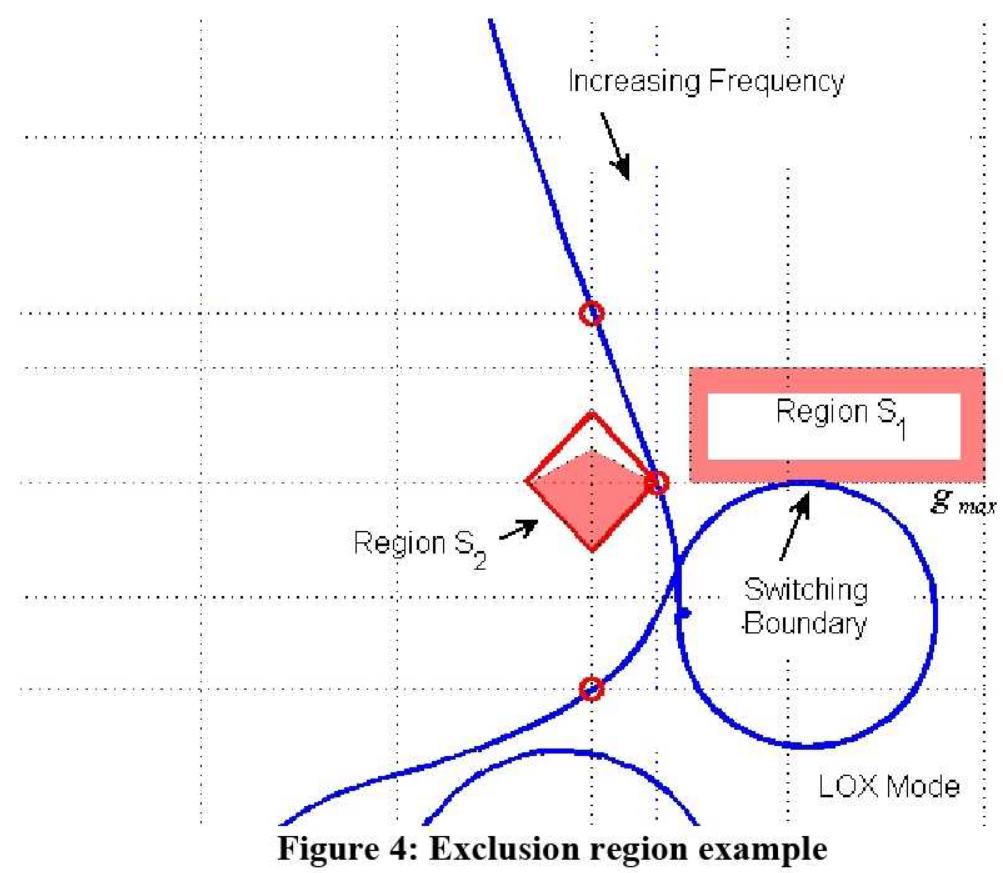

\section{Results}

The proposed algorithm is employed to determine a damping profile where the varied parameters $\theta_{1}, \theta_{2}$ are the LOX and LH2 damping ratios, respectively. The system is most sensitive to LOX damping due to the LOX mode frequency and the large slosh mass, but both tank profiles can be optimized such that stability is maintained with minimum baffle mass. The use of the present technique allows rapid convergence to a near-exact solution. Typically, $\epsilon_{i}=2 \mathrm{E}-5$ for an attained resolution in the damping ratio of approximately $1 \mathrm{E}-4$. This is more than sufficient to specify for the purposes of baffle design.

Algorithm convergence typically occurs in only 20-30 iterations and occupies about 30-60 seconds of computer time per trajectory time point on a modern (ca. 2008) desktop computer. The rapid computation cycle allows the determination of the optimum damping ratios with high resolution in the trajectory, typically every second. The use of a full-order linear model is employed so that all relevant flexible mode coupling effects are considered.

While the region $S_{1}$ in Figure 4 is arbitrarily defined, it is intuitive that regions may also be defined with respect to stability margin objectives, so that the optimized response does not pass in the vicinity of a minimum gain or phase margin. The region $S_{2}$ shown in Figure 4 is such an example.

Several points along the trajectory are considered, and the solution is determined at each point. The solution set, applied to a series of linear systems representing the open-loop controller-plant dynamics, yields a series of frequency response curves that ideally do not intersect the exclusion regions $S_{i}$. An example of a solution set for upper-stage flight is shown in Figure 6. 


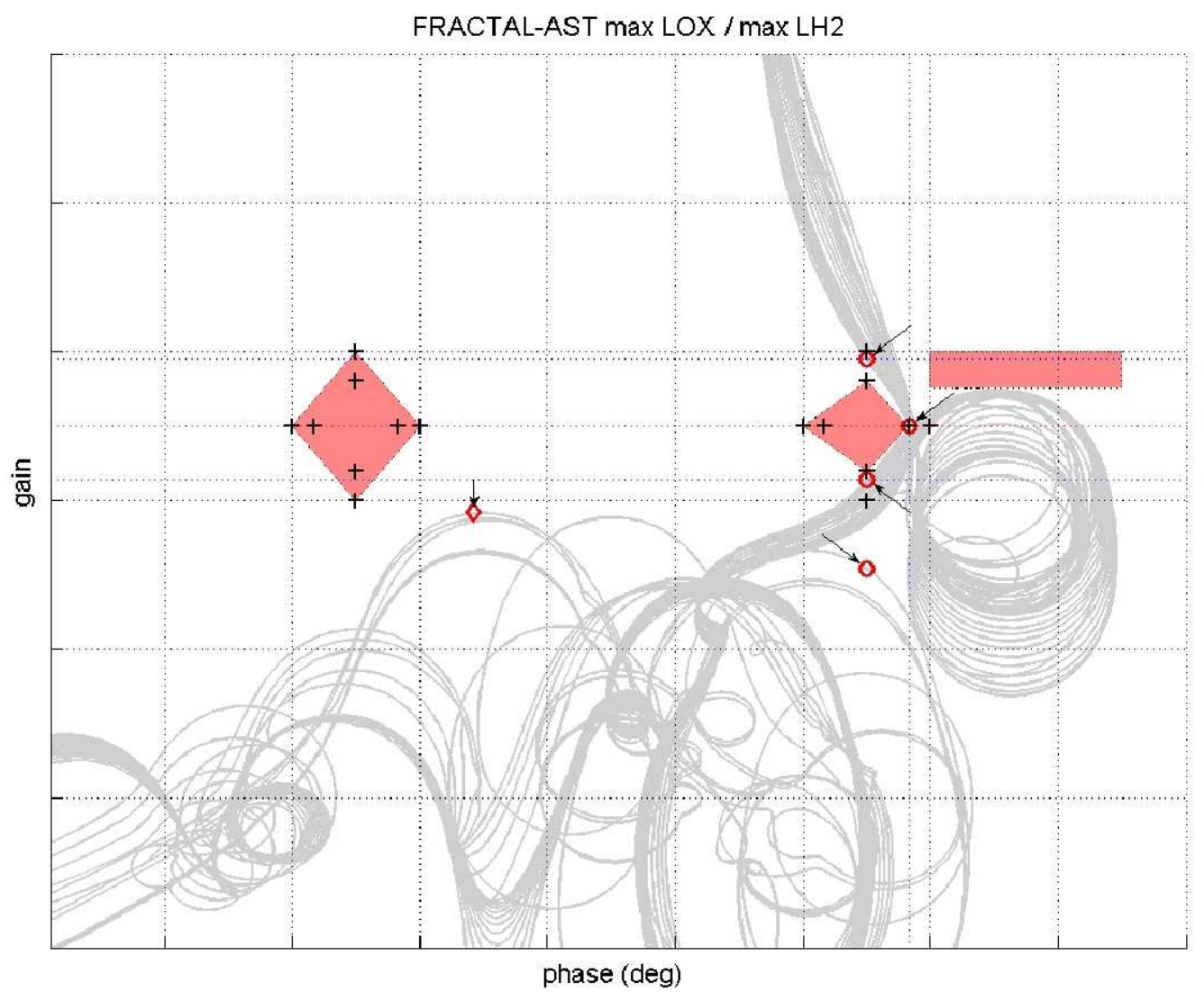

Figure 5: Upper-stage trajectory solution set

After suitable postprocessing, a minimum damping requirements table can be generated that catalogues the minimum acceptable damping values $\theta_{1}, \theta_{2}$ that achieve the prescribed response characteristics with respect to the time evolution of the trajectory. This data is delivered to structures disciplines for use in baffle design. The time axis of the trajectory may be directly transcribed to nominal propellant and oxidizer liquid levels and used to generate a requirements table with respect to the fluid level in each tank. A tank liquid level versus damping requirements table is graphically depicted in Figure 6.

Other extensions of the proposed algorithm have been implemented, including optimization of parameters in the presence of other modeling uncertainties. The approach relies on the speed of computing the linear system dynamics to systematically determine the required parameters that meet stability margin requirements while dispersing the remainder of the system parameters using Monte Carlo methods. Since the geometric criteria is binary, the algorithm need not compute the full set of random draws after the failure of a single test case, greatly reducing the overhead associated with the Monte Carlo method. Finally, the number of Monte-Carlo runs per test case can be increased using the relation such as

$$
n=n_{0}+\left|\gamma\|\boldsymbol{\epsilon}\|^{-1}\right|
$$

where $\gamma$ is a gain parameter and $n$ is bounded by $n_{\max }$, so that the full number of dispersions is not exercised until the solution is very near the converged value. 


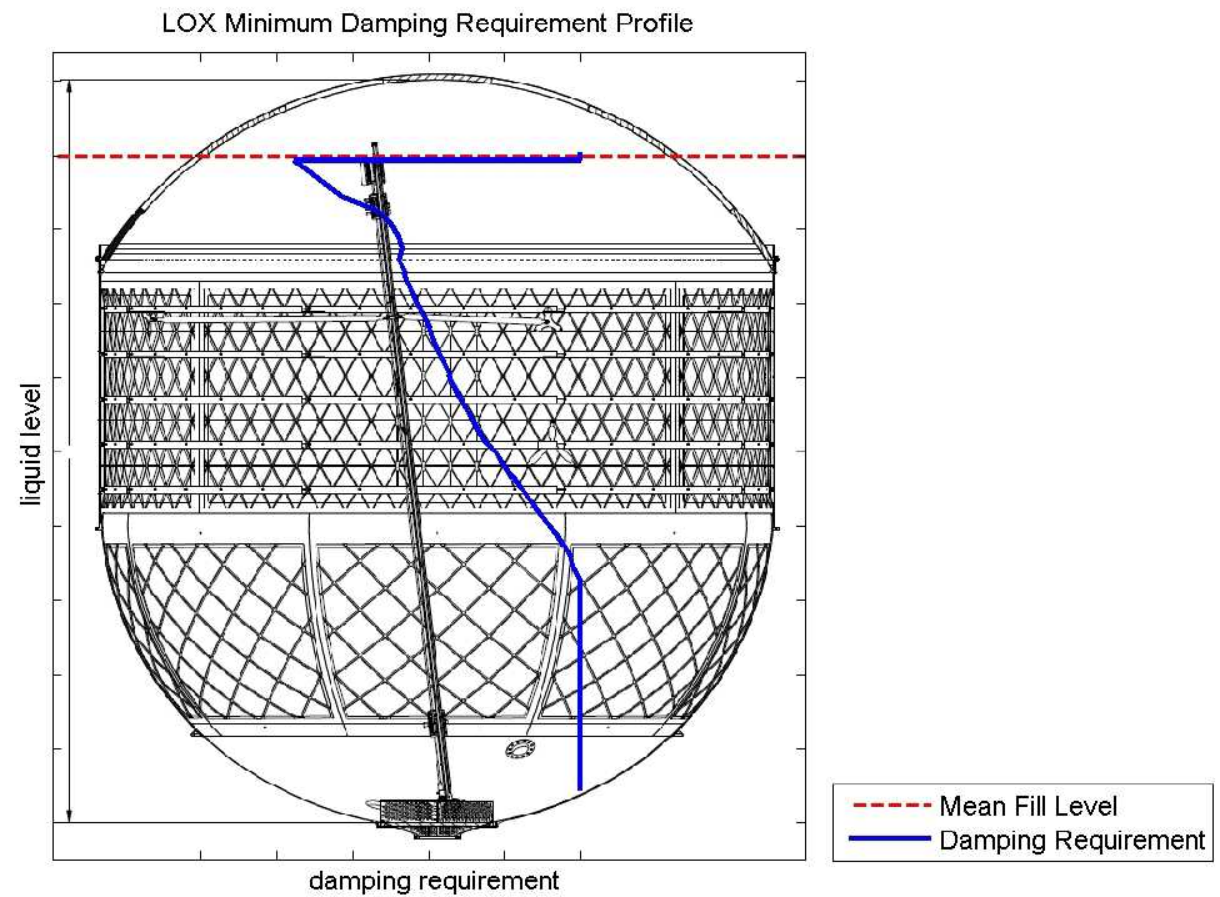

Figure 6: Minimum damping requirements example

\section{Conclusions}

The proposed method has been demonstrated to be effective in rapidly producing a set of minimum slosh damping requirements that satisfy flight control system performance objectives. The binary search algorithm capitalizes on known magnitude-phase response trends in order to construct pass-fail criteria and allow a solution to be determined much faster than with a direct search or a manual method. This technique has been used in other other parametric optimization and senstivity studies and will likely continue to reduce labor expenditure and enhance fidelity in the design and analysis of the Ares I and similar series of vehicles.

\section{Acknowledgments}

This work was prepared by bd Systems, an SAIC company, for the NASA Marshall Space Flight Center under contract to MSFC (NNM06AA01Z). 


\section{References}

${ }^{1}$ Greensite, A., "Analysis and Design of Space Vehicle Flight Control Systems, Volume I - Short Period Dynamics," NASA CR-820, 1967.

²Garner, D., "Control Theory Handbook," NASA TM-X-53036, 1964.

${ }^{3}$ Abramson, H. "The Dynamic Behavior of Liquids in Moving Containers," NASA SP-106, 1966.

${ }^{4}$ Propellant Slosh Loads, NASA Space Vehicle Design Criteria (Structures), NASA SP-8009, August 1968.

${ }^{5}$ Ringland, R., "Dynamic Stability Of Space Vehicles, Volume X - Exit Stability,” NASA CR-944, 1968.

${ }^{6}$ Bauer, H.F., "Propellant Sloshing Problems of Saturn Test Flight SA-1 (U)," NASA TM-X-50497, 1962.

${ }^{7}$ Slosh Suppression, NASA Space Vehicle Design Criteria (Structures), NASA SP-8031, May 1969.

${ }^{8}$ Frosch, J. and Vallely, D., "Saturn AS-501/S-IC Flight Control System Design," J. Spacecraft, Vol. 4, No. 8, 1967, p. 1003-1009.

9Orr, J. "FRACTAL Linear Model Dynamics," BD TCD20090535A, NASA MSFC/EV41, April 29, 2009. 